\title{
Slope failure analysis using the random material point method
}

\author{
B. WANG*, M. A. HICKS* and P. J. VARDON*
}

\begin{abstract}
The random material point method (RMPM), which combines random field theory and the material point method (MPM), is proposed. It differs from the random finite-element method (RFEM), by assigning random field (cell) values to material points that are free to move relative to the computational grid rather than to Gauss points in a conventional finite-element mesh. The importance of considering the effects of both large deformations and the spatial variability of soil strength properties in slope stability analyses is highlighted, by comparing RMPM solutions with RFEM and deterministic MPM solutions for an idealised strain-softening clay slope characterised by a spatially varying undrained shear strength. The risks posed by potential slides are quantified by the extent of retrogressive failure that is, due to the tendency for secondary failures to be triggered by the removal of support from the remaining soil mass caused by the initial failure. The results show that RMPM provides a much wider range of solutions, in general increasing the volume of material in the failure compared with the RFEM solutions, which are usually limited to the initial slide. Moreover, the anisotropic nature of soil heterogeneity is shown to have a significant influence on the nature and extent of failure.
\end{abstract}

KEYWORDS: landslides; numerical modelling; statistical analysis

ICE Publishing: all rights reserved

\author{
NOTATION \\ a acceleration \\ $c$ cohesion \\ $c_{0}, c_{\mathrm{p}}$ initial (peak) cohesion \\ $c_{\mathrm{r}}$ residual cohesion \\ $c_{\mathrm{u}} \quad$ undrained shear strength \\ $E$ Young's modulus \\ $f_{\mathrm{n}}$ nodal normal force \\ $f_{\mathrm{t}}$ nodal tangential force \\ $H$ hardening/softening modulus \\ I identity matrix \\ $i$ node numbering \\ M mass matrix \\ $m$ material mass \\ $N$ shape function \\ $N_{\mathrm{m}}$ number of material points surrounding a node \\ $N_{\mathrm{n}} \quad$ number of grid nodes around a material point \\ $p$ material point numbering \\ $r$ sliding distance \\ $t$ time \\ v velocity \\ $V$ coefficient of variation \\ $\boldsymbol{x}$ location \\ $z$ depth below slope crest \\ r soil unit weight \\ $\Delta t$ time step \\ $\varepsilon_{\mathrm{p}}$ plastic strain \\ $\bar{\varepsilon}_{\mathrm{p}} \quad$ plastic shear strain invariant \\ $\bar{\varepsilon}_{\mathrm{pr}}$ plastic shear strain invariant at the onset of residual strength \\ $\theta_{\mathrm{h}}$ horizontal scale of fluctuation \\ $\theta_{\mathrm{v}} \quad$ vertical scale of fluctuation \\ $\mu$ mean \\ $\mu_{\mathrm{f}}$ frictional coefficient \\ $\sigma$ standard deviation \\ $v$ Poisson's ratio
}

Manuscript received 28 January 2016; first decision 25 April 2016; accepted 3 May 2016.

Published online at www.geotechniqueletters.com on 10 June 2016.

*Section of Geo-Engineering, Faculty of Civil Engineering and Geosciences, Delft University of Technology, Delft, The Netherlands.

\section{INTRODUCTION}

Soils exhibit spatial variability of material properties due to variations in density, particle size distribution, minerology and stress history. By considering heterogeneity within a statistical framework, it is evident that slopes can exhibit a variety of failure mechanisms due to the spatial distribution of the weaker zones. For example, by using the random finite-element method (RFEM), it has been shown that failure mechanisms tend to follow the path of least resistance (Hicks \& Samy, 2002; Li et al., 2015), and that the consequences of failure - for example, slide volume, are closely related to the category of failure mode (Hicks \& Spencer, 2010; Hicks et al., 2014). RFEM combines random field theory, for modelling spatial variability, with the finite-element method (FEM), for modelling geotechnical response, within a Monte Carlo simulation process (Fenton $\&$ Griffiths, 2008). For this purpose, finite-element analyses are often limited to small deformation problems, in order to avoid excessive mesh distortions. In contrast, the material point method (MPM) (Sulsky et al., 1994; Sulsky \& Schreyer, 1996) has proven to be a useful FEM variant in capturing geotechnical failure mechanisms involving large deformations (Wieckowski et al., 1999; Al-Kafaji, 2013; Bandara \& Soga, 2015; Wang et al., 2016).

By combining random field theory and MPM, this paper proposes a new technique to investigate the influence of heterogeneity on slope failure mechanisms involving large deformations, including secondary failure mechanisms and failure consequences. This technique is called the 'random material point method' (RMPM).

\section{THEORETICAL FORMULATIONS \\ MPM}

MPM uses two types of discretisation: $(a)$ a background mesh for computation, which is regularly reset to limit mesh distortions and $(b)$ a set of material points representing the material, which store all state variables and are allowed to move freely through the mesh. There are three 


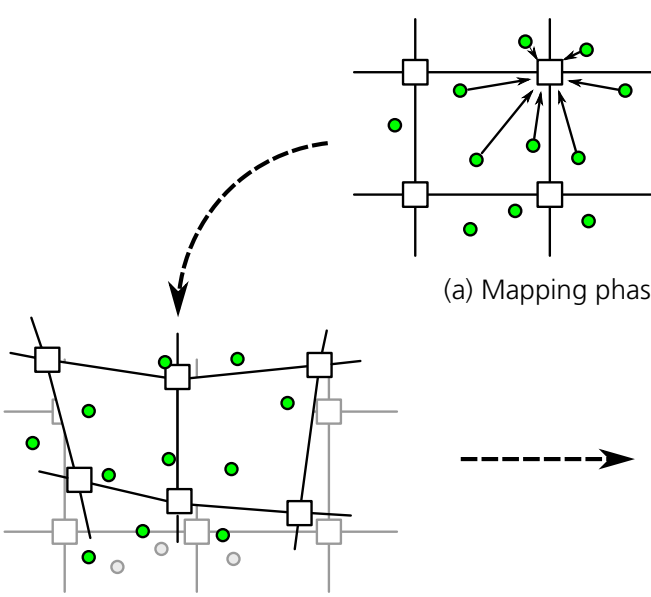

(b) UL-FEM phase

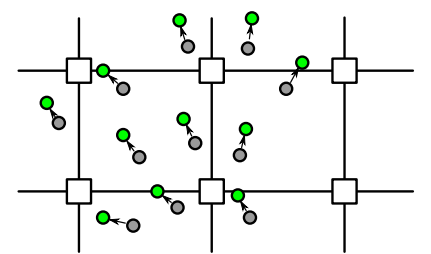

(c) Convection phase

Fig. 1. Computational cycle of MPM: (a) A set of material points representing the material, overlaid on a background computational mesh. Material properties, constitutive models and other state information are assigned to, and stored only on, the material points. Information is transferred to the nodes of the background mesh for computational purposes. (b) The equations of motion are solved on the background mesh, utilising the updated Lagrangian FEM. (c) The state of the material points is updated, and the background mesh reset

computational phases, as illustrated in Fig. 1 and summarised as follows.

In the mapping phase, state variable information is transferred from the material points to the background mesh nodes - for example

$$
\boldsymbol{v}_{i}=\sum_{p=1}^{N_{\mathrm{m}}} \frac{N_{i}\left(\boldsymbol{x}_{p}\right) m_{p} \boldsymbol{v}_{p}}{m_{i}}
$$

where subscripts $i$ and $p$ refer to the nodal and material point numbering, respectively; $m$ and $\boldsymbol{v}$ are the material mass and velocity; $N(\boldsymbol{x})$ are the shape functions at location $\boldsymbol{x}$ and $N_{\mathrm{m}}$ is the number of material points surrounding node $i$.

The updated Lagrangian FEM phase follows standard FEM procedures to solve the governing equations, although a major algorithmic difference is in the use of the material points to perform numerical integration - for example

$$
\mathbf{M}_{i}=\sum_{p=1}^{N_{\mathrm{m}}} N_{i}\left(\boldsymbol{x}_{p}\right) m_{p} \mathbf{I}
$$

where $\mathbf{M}_{i}$ is the contribution to the mass matrix from node $i$ and $\mathbf{I}$ is an identity matrix. The nodal accelerations are then solved for time $t+\Delta t$

The convection stage involves mapping and updating of kinematic variables back to the material points - that is

$$
\begin{aligned}
& \boldsymbol{a}_{p}^{t+\Delta t}=\sum_{i=1}^{N_{\mathrm{n}}} \boldsymbol{a}_{i}^{t+\Delta t} N_{i}\left(\boldsymbol{x}_{p}^{t}\right) \\
& \boldsymbol{v}_{p}^{t+\Delta t}=\boldsymbol{v}_{p}^{t}+\boldsymbol{a}_{p}^{t+\Delta t} \Delta t \\
& \boldsymbol{x}_{p}^{t+\Delta t}=\boldsymbol{x}_{p}^{t}+\boldsymbol{v}_{p}^{t+\Delta t} \Delta t
\end{aligned}
$$

where $N_{\mathrm{n}}$ is the number of grid nodes providing support to the material point.

Depending on how the governing equations are integrated and solved in time, MPM is either categorised as implicit (Wang et al., 2013, 2016) or explicit (Sulsky et al., 1994). However, the key aspects of both formulations are the same, including the techniques for treating boundaries, damping and so on. An explicit version of the code has been adopted here, based on Sulsky \& Schreyer (1996).

\section{Random field theory}

Random fields are numerically generated predictions of the spatial variability of a property over the problem domain. These are based on $(a)$ an assumed probability density function, characterised by the mean property value $\mu$ and standard deviation $\sigma$, in which $V=\sigma / \mu$ is the coefficient of variation (Fig. 2(b)) and (b) an assumed spatial correlation function, characterised by vertical and horizontal scales of fluctuation, $\theta_{\mathrm{v}}$ and $\theta_{\mathrm{h}}$, respectively, defining the distance over which property values are significantly correlated (Fig. 2(a)).

This paper uses local average subdivision to generate random fields of local averages that are assigned to random field cells (Fenton \& Vanmarcke, 1990). RFEM involves multiple realisations of the problem to be solved, in which each realisation involves generating a random field of the material property, mapping cell values from the random field onto the FEM mesh at the Gauss point level, and

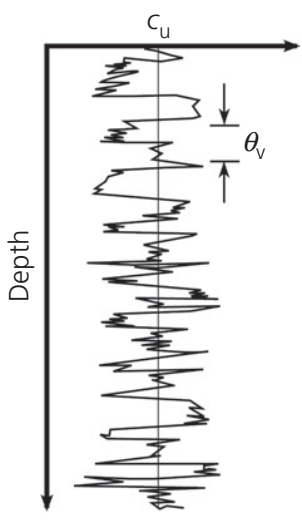

(a)

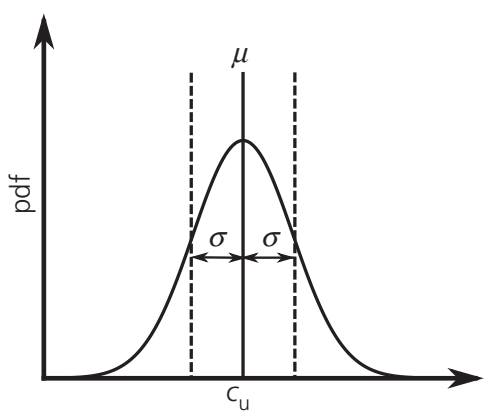

(b)
Fig. 2. Statistical measures of $c_{\mathrm{u}}$ variability (after Hicks \& Samy, 2002): (a) variation of $c_{u}$ with depth, (b) probability density function of $c_{\mathrm{u}}$ 


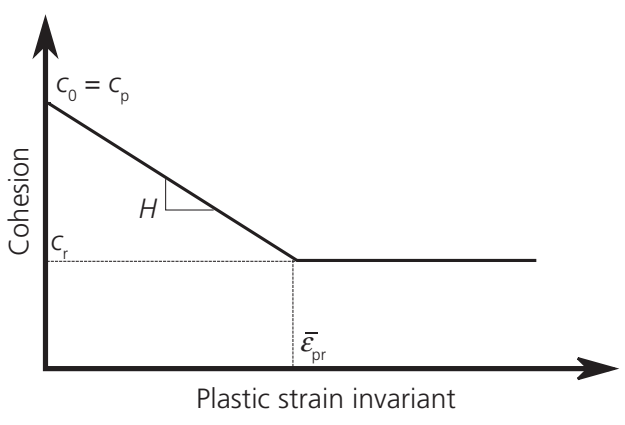

Fig. 3. Sketch of cohesion-softening model

analysing the problem deterministically by finite elements (Hicks \& Spencer, 2010). In contrast, the random field is mapped to the problem domain at the material point level in RMPM.

In both RFEM and RMPM, multiple realisations are analysed for the same set of input statistics (i.e. $\mu, \sigma, \theta_{\mathrm{v}}, \theta_{\mathrm{h}}$ ), in order to obtain a distribution of possible responses and their relative likelihood.

\section{Cohesion-softening model}

For illustrative purposes, a simple cohesion-softening Von Mises material model has been used herein. Figure 3 shows that the strength decreases proportionally to the plastic strain invariant in the softening stage - that is

$$
\begin{aligned}
& c\left(\bar{\varepsilon}_{\mathrm{p}}\right)=c_{0}+H \bar{\varepsilon}_{\mathrm{p}}, \quad \bar{\varepsilon}_{\mathrm{p}}<\bar{\varepsilon}_{\mathrm{pr}} \\
& c\left(\bar{\varepsilon}_{\mathrm{p}}\right)=c_{\mathrm{r}}, \quad \bar{\varepsilon}_{\mathrm{p}}>\bar{\varepsilon}_{\mathrm{pr}}
\end{aligned}
$$

where $c$ is the (undrained) cohesion; $c_{0}$ and $c_{\mathrm{r}}$ are the initial (i.e. peak) and residual cohesions, respectively; $\bar{\varepsilon}_{\mathrm{p}}=\sqrt{(2 / 3) \varepsilon_{\mathrm{p}}: \varepsilon_{\mathrm{p}}}$ is the plastic shear strain invariant; $\bar{\varepsilon}_{\mathrm{pr}}$ is the plastic shear strain invariant at the onset of the residual strength; $\varepsilon_{\mathrm{p}}$ is the vector of plastic strains and $H$ is the hardening or softening modulus (defined as negative for softening).

\section{INFLUENCE OF SOIL HETEROGENEITY ON RETROGRESSIVE SLOPE FAILURES}

An idealised total stress boundary value problem has been analysed to provide a simple illustration of RMPM, although the same methodology can (and will in the future) be applied to coupled effective stress analyses. Figure 4 shows a $45^{\circ}, 5 \mathrm{~m}$ high, clay slope, resting on a firm base. The length from the slope crest to the left-hand boundary is $20 \mathrm{~m}$, in order to provide enough space for the development of retrogressive failures. The initial slope geometry has been discretised using 1810, four-node, quadrilateral elements, with four material points per element located at the Gauss point positions (i.e. initially, before the slope starts to deform). A frictional contact

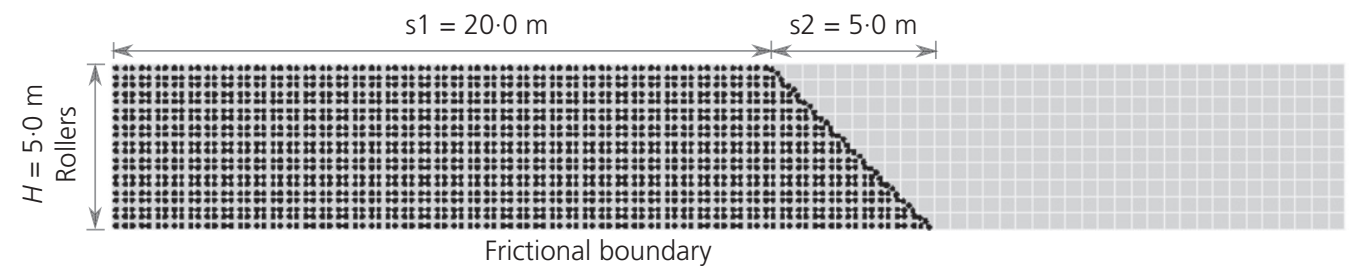

Fig. 4. Boundary conditions, background mesh and initial geometry of the clay slope. A cruder mesh than that used in the analysis is shown for reasons of clarity

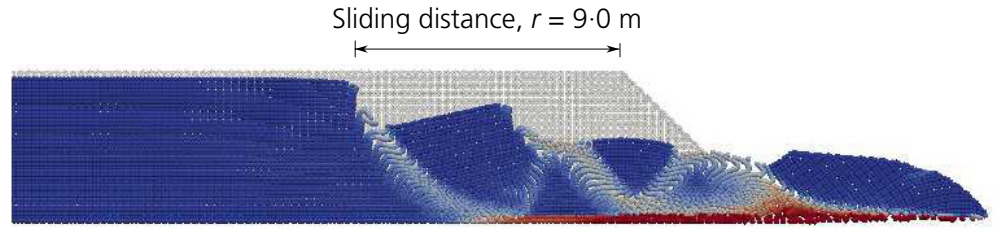

(a)

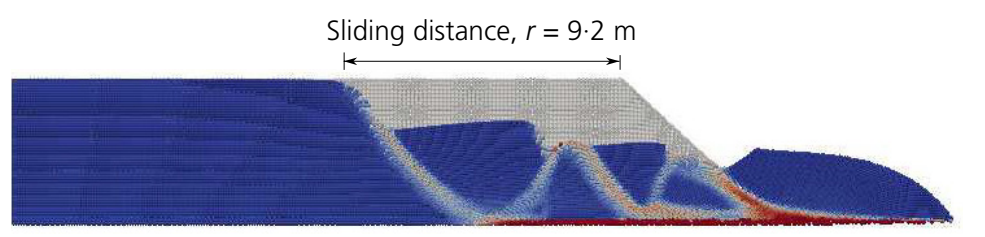

(b)

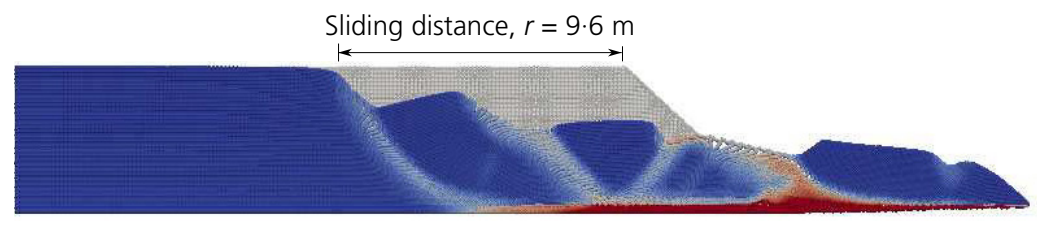

(c)
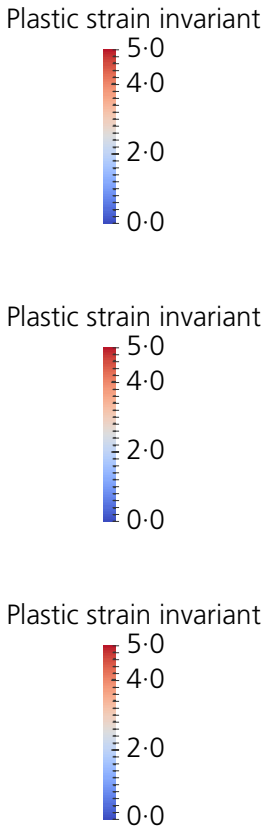

Fig. 5. Initial and final slope configurations, with contours of plastic shear strain invariant, including spatial convergence analysis: (a) Most coarse converged mesh (1810 elements, 4 material points per element), (b) Refined elements (3840 elements, 4 material points per element), (c) Refined material points (1810 elements, 16 material points per element) 


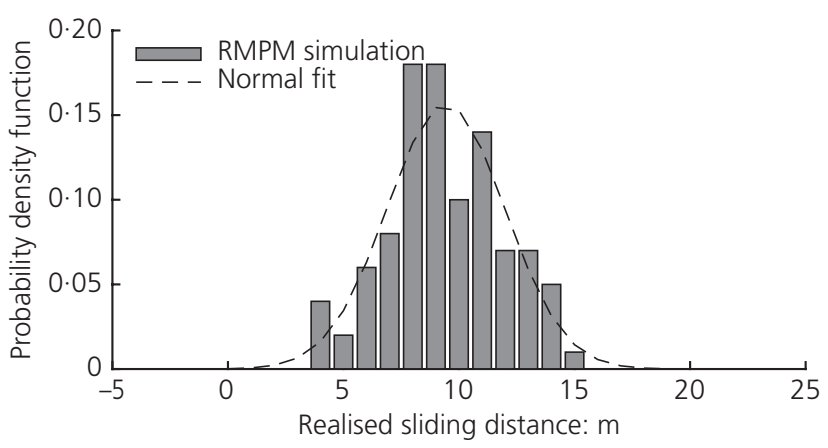

(a)

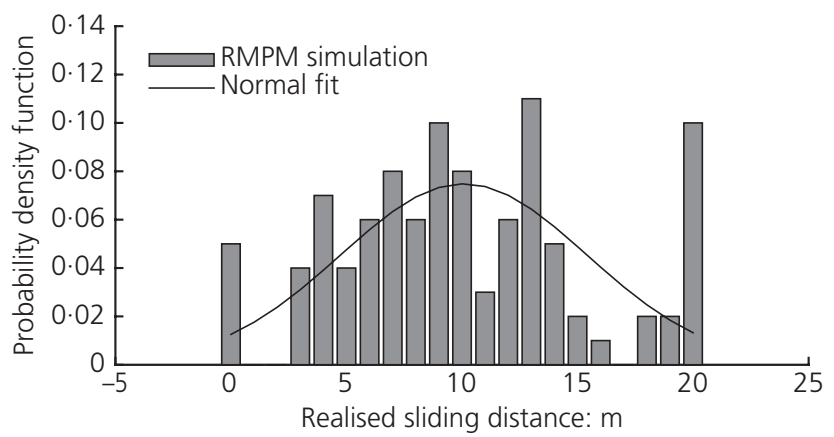

(c)

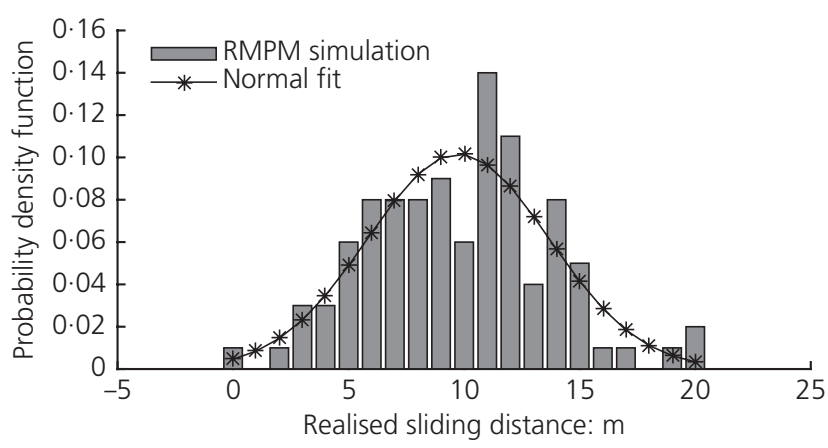

(b)

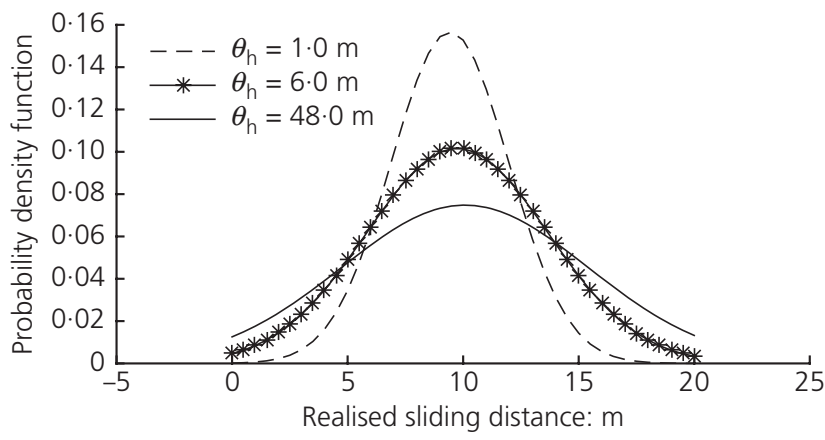

(d)

Fig. 6. Probability density functions of the realised sliding distance for the slope: (a) $\theta_{\mathrm{h}}=1.0 \mathrm{~m}$, (b) $\theta_{\mathrm{h}}=6 \cdot 0 \mathrm{~m}$, (c) $\theta_{\mathrm{h}}=48 \cdot 0 \mathrm{~m}$, (d) influence of $\theta_{\mathrm{h}}$ on PDF

between the slope base and supporting firm ground is assumed, to account for geometric interlocking between material layers, while rollers allow only vertical movement at the left-hand boundary. The frictional boundary is based on Bandara \& Soga (2015) and is defined by a maximum nodal tangential force $f_{\mathrm{t}}=\mu_{\mathrm{f}} f_{\mathrm{n}}$, where $f_{\mathrm{n}}$ is the nodal force normal to the boundary and, in this analysis, the frictional coefficient is $\mu_{\mathrm{f}}=0 \cdot 3$. The Young's modulus and Poisson's ratio of the soil are $E=1000 \mathrm{kPa}$ and $v=0 \cdot 33$, respectively. While an undrained failure implies a Poisson's ratio of 0.5 , the adopted smaller value aids numerical convergence and promotes a more realistic in-situ stress field, whereas the plastic model component dominates the failure mechanism. The peak undrained shear strength is $20 \mathrm{kPa}$, the residual strength is $4 \mathrm{kPa}$ and the hardening/softening modulus is $-50 \mathrm{kPa}$. For simplicity, the slope is initially unstable under its own weight; hence, slope failure is triggered by applying gravitational loading to generate the in-situ stresses.

Figure 5(a) shows the final slope configuration computed using a deterministic MPM analysis. For a straightforward quantification of the potential failure consequence, the sliding distance $r$, as shown in Fig. 5, is used to define how far the failure retrogresses backwards from the crest. Figures 5(b) and 5(c) investigate mesh dependency by considering refined meshes with greater numbers of elements and material points, respectively. Although, as expected, some mesh dependency is observed, the overall behaviour, number of failures and sliding distance are consistent enough to ensure the veracity of the following numerical study (which is based on the discretisation in Fig. 5(a)).

\section{RMPM against deterministic analysis}

The influence of spatial variability of undrained shear strength on slope response has been assessed by assuming a normal distribution and a coefficient of variation of 0.25 for both peak and residual shear strengths (with all other parameters being the same as for the MPM analysis). A series of RMPM analyses, each comprising 100 Monte Carlo realisations, has been conducted, for $\theta_{\mathrm{v}}=1.0 \mathrm{~m}$ and $\theta_{\mathrm{h}}=1 \cdot 0,6 \cdot 0$ and $48 \cdot 0 \mathrm{~m}$.

Figures 6(a)-6(c) show the probability density histograms of the computed sliding distance, as well as fitted normal probability density functions. The normal distribution fits the computed results of the Monte Carlo simulation reasonably well for smaller $\theta_{\mathrm{h}}$, as in Figs 6(a) and 6(b). Although Fig. 6(c) shows a less good fit due to the higher number of realisations needed for convergence with higher $\theta_{\mathrm{h}}$ (Hicks \& Samy, 2002), the number of realisations is sufficient to enable proper evaluation of trends. Moreover, the peak in the number of realisations showing a sliding distance of $20 \mathrm{~m}$ is an indication that the mesh does not extend far enough from the slope crest in some cases. The mean sliding distances for $\theta_{\mathrm{h}}=1.0,6.0$ and $48.0 \mathrm{~m}$ are $r=9.43,9.75$ and $10.07 \mathrm{~m}$, respectively, compared with $r=9.0 \mathrm{~m}$ for the deterministic MPM analysis based on the mean undrained strength. However, of greater practical significance is that the standard deviation of (i.e. the uncertainty in) $r$ increases significantly with $\theta_{\mathrm{h}}$, by a factor of around 2 for the range of

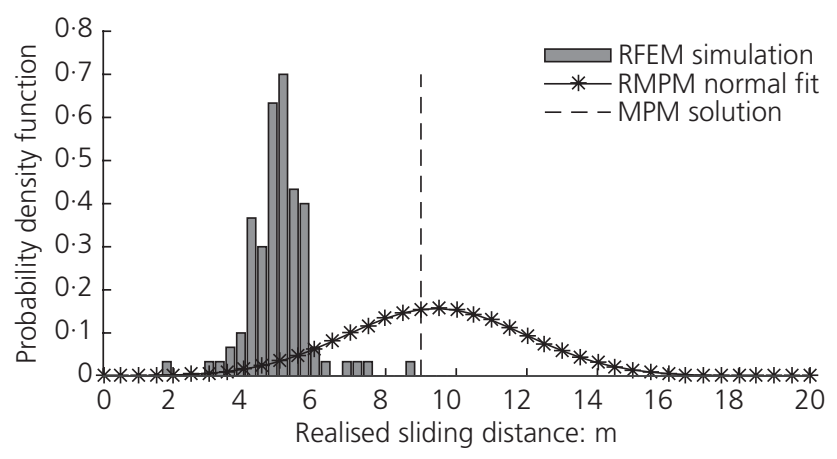

Fig. 7. Comparison of solutions for $\theta_{\mathrm{h}}=1.0 \mathrm{~m}$ 


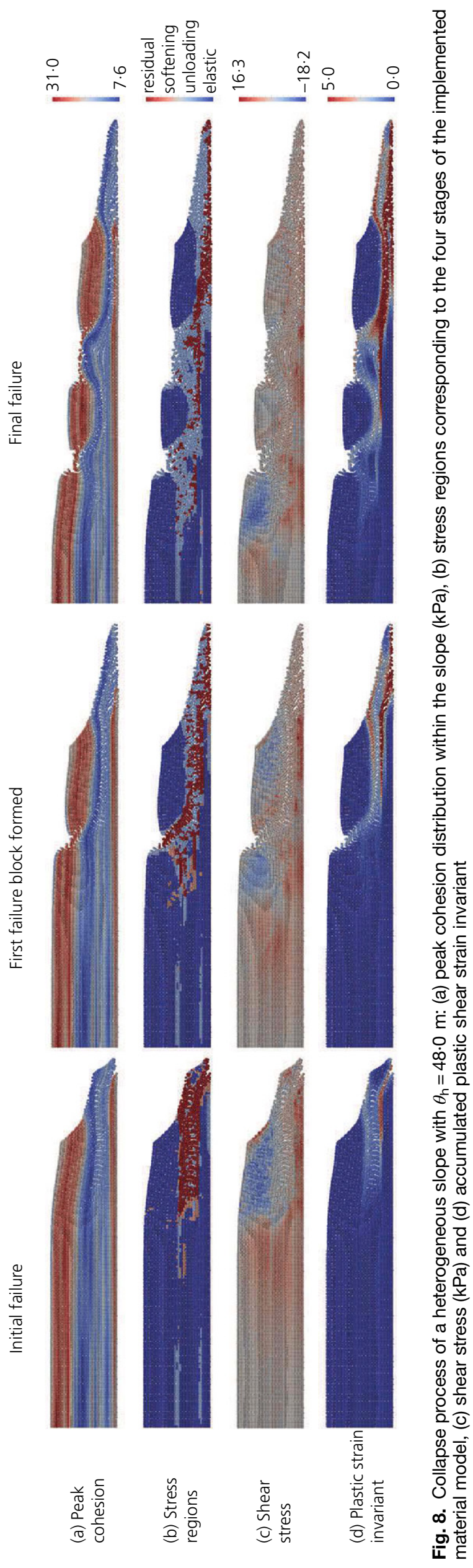

$\theta_{\mathrm{h}}$ considered. Hence, the deterministic analysis may seriously underestimate the sliding distance, giving an unconservative estimate of the risk. For the case in which $\theta_{\mathrm{h}}=48.0 \mathrm{~m}$, the spatial variability takes on a layered appearance. A possible consequence is that the slope remains stable (i.e. $r=0 \mathrm{~m}$ ) due to the presence of highstrength soil layers along the potential failure path, especially near the slope toe; conversely, the slope can slide a large distance (e.g. $r>20 \mathrm{~m}$ ) due to the presence of weak layers.

Note that some reduction of the distribution width may be possible, by constraining the spatial variability using site-specific data to condition the random fields (Lloret-Cabot et al., 2014).

\section{RMPM against RFEM}

RFEM solutions have been compared with the results of RMPM (for the same ensemble of random fields), as shown in Fig. 7 for $\theta_{\mathrm{h}}=1.0 \mathrm{~m}$. Due to the assumption of small deformations in the RFEM simulation carried out in this paper, secondary slides were generally not computed, thereby significantly reducing the sliding distance and range of solutions relative to the RMPM simulation. With RMPM, the failure is able to retrogress backwards during the slope collapse, due to the removal of support provided by soil involved in the initial failure.

\section{Failure process of heterogeneous slopes}

Figure 8 shows the three stages in the failure evolution for a typical realisation in which $\theta_{\mathrm{h}}=48.0 \mathrm{~m}$ : the initial failure, the first failure block formed and the final configuration. Figure 8(a) shows the random field of peak undrained shear strength, with blue and red representing weak and strong zones, respectively. As the soil self-weight is applied, the shear stresses start to build up near the slope toe. However, the failure initiates slightly above the toe where the slope stability number $\left(c_{\mathrm{u}} / \gamma z\right)$ is lower. Hence, the failure mechanism avoids the stronger zone in seeking out the path of least resistance and fails along the base of the weaker soil.

Figure $8(\mathrm{~b})$ depicts the stages of the constitutive model governing the behaviour of the material points within the slope. Blue, light blue, orange and red in the figure indicate elastic, elastic unloading, softening and residual stages of the model, respectively. It is seen that the material points within a developing shear band are mostly governed by the residual cohesion, whereas the soils within previous shear bands mostly experience elastic unloading as a new shear band is being formed. Figure 8(d) shows that the largest plastic shear strain invariant contours propagate mainly horizontally, forming the basal line of the global failure. Due to the removal of support from the soil at the backscarp of the first slide, the failure of the backscarp is triggered and the second failure block is formed, with deformation continuing until the final equilibrium state.

The influence of anisotropy (of the heterogeneity) on the slope failure modes is illustrated in Fig. 9, where typical final slope configurations for $\theta_{\mathrm{h}}=1.0$ and $6.0 \mathrm{~m}$ are shown. The contours of plastic shear strain invariant, in Figs 8(d), 9(b) and 9(d), demonstrate that more soil wedges tend to form for smaller $\theta_{\mathrm{h}}$, due to it being easier for failure paths to avoid stronger zones.

\section{CONCLUSION}

The random material point method (RMPM) has been proposed. In contrast to RFEM, which assigns random field (cell) values to Gauss points within a finite-element mesh, 


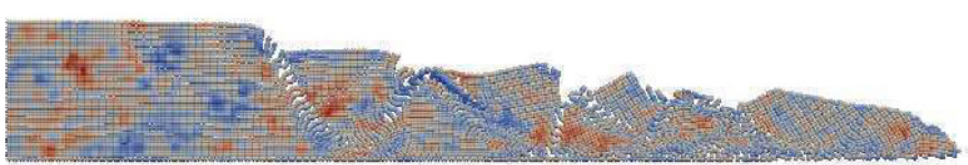

(a)

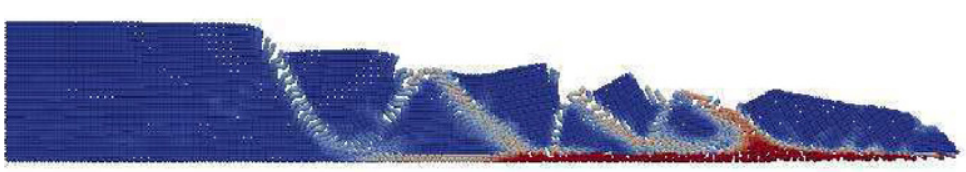

(b)

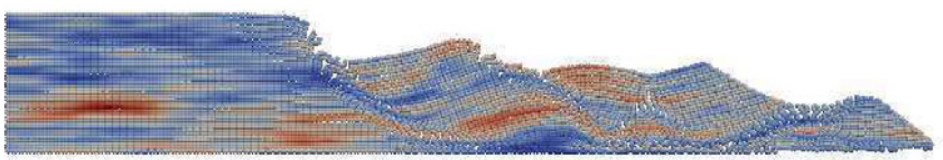

(c)

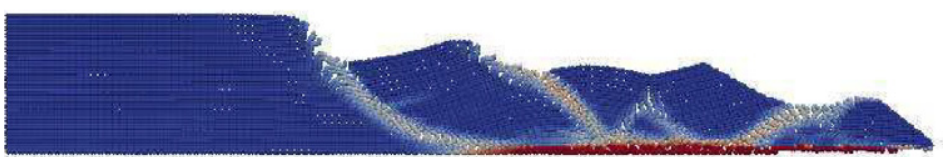

(d)

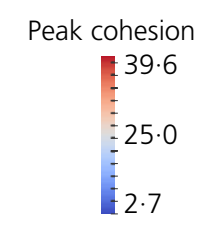

Plastic strain invariant

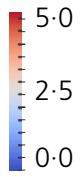

Peak cohesion

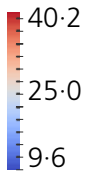

Plastic strain invariant

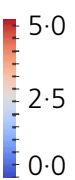

Fig. 9. Typical final configurations for $\theta_{\mathrm{h}}=1.0$ and $6.0 \mathrm{~m}:(\mathrm{a}) \theta_{\mathrm{h}}=1.0 \mathrm{~m}$, random field of peak cohesion (kPa), (b) $\theta_{\mathrm{h}}=1 \cdot 0 \mathrm{~m}$, plastic shear strain invariant contours, (c) $\theta_{\mathrm{h}}=6.0 \mathrm{~m}$, random field of peak cohesion $(\mathrm{kPa}),(\mathrm{d}) \theta_{\mathrm{h}}=6.0 \mathrm{~m}$, plastic shear strain invariant contours

RMPM assigns random field values to the so-called material points that are able to move through the computational grid. This grid is regularly reset, to avoid the excessive mesh distortions that can occur in large deformation finiteelement analyses.

The potential of RMPM in geotechnical analysis has been simply demonstrated for an idealised slope in a strainsoftening soil. Large deformations and heterogeneity were both shown to have a significant effect on the initiation and evolution of the slope failure mechanism; in particular, on secondary failures and the extent of failure retrogression. By equating the potential failure consequence to the sliding distance (i.e. extent), deterministic and/or small strain analyses are shown to, in general, yield unconservative results and underestimate the risk.

\section{ACKNOWLEDGEMENTS}

The authors acknowledge the China Scholarship Council and the Geo-Engineering Section of Delft University of Technology for their financial support of the first author, as well as the partial support by a Marie Curie Career Integration Grant (no. 333177) for the third author.

\section{REFERENCES}

Al-Kafaji, I. K. J. (2013). Formulation of a dynamic material point method (MPM) for geomechanical problems. PhD thesis, Stuttgart University, Stuttgart, Germany.

Bandara, S. \& Soga, K. (2015). Coupling of soil deformation and pore fluid flow using material point method. Comput. Geotech. 63, 199-214.

Fenton, G. A. \& Griffiths, D. V. (2008). Risk assessment in geotechnical engineering. New York, NY, USA: John Wiley and Sons.

Fenton, G. A. \& Vanmarcke, E. H. (1990). Simulation of random fields via local average subdivision. J. Engng Mech. 116, No. 8 , 1733-1749.
Hicks, M. A. \& Samy, K. (2002). Influence of heterogeneity on undrained clay slope stability. Q. J. Engng Geol. Hydrogeol. 35, No. 1, 41-49.

Hicks, M. A. \& Spencer, W. A. (2010). Influence of heterogeneity on the reliability and failure of a long 3D slope. Comput. Geotech. 37, No. 7, 948-955.

Hicks, M. A., Nuttall, J. D. \& Chen, J. (2014). Influence of heterogeneity on $3 \mathrm{D}$ slope reliability and failure consequence. Comput. Geotech. 61, 198-208.

Li, Y., Hicks, M. A. \& Nuttall, J. D. (2015). Comparative analyses of slope reliability in 3D. Engng Geol. 196, 12-23.

Lloret-Cabot, M., Fenton, G. A. \& Hicks, M. A. (2014). On the estimation of scale of fluctuation in geostatistics. Georisk, Assess. Manage. Risk Engrd Syst. Geohazards 8, No. 2, 129-140.

Sulsky, D. \& Schreyer, H. L. (1996). Axisymmetric form of the material point method with applications to upsetting and Taylor impact problems. Comput. Methods Appl. Mech. Engng 139, No. 1, 409-429.

Sulsky, D., Chen, Z. \& Schreyer, H. L. (1994). A particle method for history-dependent materials. Comput. Methods Appl. Mech. Engng 118, No. 1, 179-196.

Wang, B., Vardon, P. J. \& Hicks, M. A. (2013). Implementation of a quasi-static material point method for geotechnical applications. 3rd international symposium on computational geomechanics, Krakow, Poland, pp. 305-313.

Wang, B., Vardon, P. J., Hicks, M. A. \& Chen, Z. (2016). Development of an implicit material point method for geotechnical applications. Comput. Geotech. 71, 159-167.

Wieckowski, Z., Youn, S. K. \& Yeon, J. H. (1999). A particle-in-cell solution to the silo discharging problem. Int. J. Numer. Methods Engng 45, No. 9, 1203-1225.

\section{WHAT DO YOU THINK?}

To discuss this paper, please email up to 500 words to the editor at journals@ice.org.uk. Your contribution will be forwarded to the author(s) for a reply and, if considered appropriate by the editorial panel, will be published as a discussion. 